\title{
Asymmetries in explosive strength following anterior cruciate ligament reconstruction
}

\author{
Olivera M. Knezevic ${ }^{\text {a,b }}$, Dragan M. Mirkov ${ }^{\text {b }}$, Marko Kadija ${ }^{\text {c }}$, Aleksandar Nedeljkovic ${ }^{\text {b }}$, Slobodan Jaric ${ }^{\text {d,e,* }}$ \\ a University of Belgrade, Institute for Medical Research, Department of Neurophysiology, Belgrade, Serbia \\ b University of Belgrade, Faculty of Sport and Physical Education, The Research Centre, Belgrade, Serbia \\ c Clinical Centre of Serbia, Institute for Orthopaedic Surgery and Traumatology, Belgrade, Serbia \\ ${ }^{\mathrm{d}}$ University of Delaware, Department of Kinesiology and Applied Physiology, Newark, DE, USA \\ e University of Delaware, Biomechanics and Movement Science Graduate Program, Newark, DE, USA
}

\section{A R T I C L E I N F O}

\section{Article history:}

Received 7 April 2014

Received in revised form 30 May 2014

Accepted 21 July 2014

\section{Keywords:}

ACL

Strength

Rate of force development

Quadriceps

Hamstrings

\begin{abstract}
A B S T R A C T
Background: Despite its apparent functional importance, there is a general lack of data regarding the time-related changes in explosive strength and the corresponding side-to-side asymmetries in individuals recovering from an ACL reconstruction (ACLR). The present study was designed to assess changes in the maximum and explosive strength of the quadriceps and hamstring muscles in athletes recovering from an ACLR.

Methods: Twenty male athletes with an ACL injury completed a standard isometric testing protocol pre-ACLR, four and six months post-ACLR. In addition to the maximum strength $\left(F_{\max }\right)$, the explosive strength of quadriceps and hamstrings was assessed through four variables derived from the slope of the force-time curves over various time intervals $\left(R F D_{\text {max }}, R_{50}, R_{150}\right.$ and $R_{25} D_{250}$ ). Side-to-side asymmetries were calculated relative to post-ACLR measures of the uninvolved leg ("standard" asymmetries), and relative to pre-ACLR value of the uninvolved leg ("real" asymmetries).

Results: Pre-ACLR asymmetries in quadriceps RFD (average 26\%) were already larger than in $F_{\max }(14 \%)$ $(\mathrm{p}<0.05)$. Six months post-ACLR real asymmetries in RFD variables (33-39\%) were larger than the corresponding standard asymmetries $(26-28 \% ; \mathrm{p}<0.01)$. Average asymmetries in hamstrings' RFD and $\mathrm{F}_{\max }$ were $10 \%, 25 \%$ and $15 \%$ for pre-ACLR and two post-ACLR sessions, respectively (all p >0.05).

Conclusions: In addition to the maximum strength, the indices of explosive strength should also be included in monitoring recovery of muscle function following an ACLR. Furthermore, pre-injury/reconstruction values should be used for the post-ACLR side-to-side comparisons, providing a more valid criterion regarding the muscle recovery and readiness for a return to sports.
\end{abstract}

(c) 2014 Elsevier B.V. All rights reserved.

\section{Introduction}

Evaluation of the quadriceps and hamstrings' maximum strength is of profound importance in monitoring recovery following an ACL reconstruction (ACLR) [4-7]. Among a number of methods applied to the assessment and monitoring of the muscle strength following an ACLR has been the standard isometric test based on the maximum voluntary contraction of the tested muscle [6,8-10]. The routinely recorded dependent variable that depicts the maximum strength has been the maximum force $\left(\mathrm{F}_{\max }\right)$, typically achieved over three-five $\mathrm{s}$ of a sustained maximum contraction $[11,12]$.

In addition to the maximum strength, the so-called explosive strength (i.e., the ability to quickly exert high muscle force $[13,14]$ )

\footnotetext{
* Corresponding author at: Department of Kinesiology and Applied Physiology, University of Delaware, Rust Arena, Rm. 143, 541 South College Avenue, Newark, DE 19716, USA. Tel.: +1 302 8316174; fax: +1 3028313693.

E-mail address: jaric@udel.edu (S. Jaric).
}

has been considered an important functional property of tested muscles. Explosive strength has been typically assessed from the slope of the force-time curve as the rate of force development (RFD) at various time intervals from the onset of the muscle contraction [13]. It has been previously demonstrated that the RFD could be influenced by different neurophysiological mechanisms at the early $(<100 \mathrm{~ms})$ and late phase $(>100 \mathrm{~ms})$ of the isometric contractions [15]. Specifically, the indices of explosive strength that obtained from the early phase of force development may predominantly depend upon the level of neural excitation. Note that a diminished quadriceps control has been observed in people with ACL deficiency [1]. Namely, loss of afferent feedback from knee joint structures leads to suppressed feedback from the ACL to gamma motor neurons and results in chronic suppression of recruitment of high-threshold motor units during voluntary contraction of the quadriceps $[2,3]$. Conversely, the same indices obtained from the later phase may be more dependent upon the muscle contractile mechanisms $[13,16]$. A number of studies have shown that the ability to quickly exert a high level of muscle force should be more important 
for maximizing movement performance than the maximum strength, particularly in the movement tasks of limited duration [13,17-19] where quick and forceful muscle contractions are needed $[16,19,20]$, such as in athletic activities like jumping and sprinting [14,21]. A high level of explosive strength may be equally important when stabilizing the posture during standing, locomotion, or in response to mechanical perturbations $[22,23]$.

Despite its apparent functional importance, there is a general lack of data regarding the time-related changes in the explosive strength and the corresponding side-to-side asymmetries in individuals recovering following an ACLR. Some aspects of the measures in monitoring recovery following musculoskeletal injuries have only recently been addressed $[13,18,24]$. Thus, the indices of explosive strength were introduced for the purpose of a predisposing risk factor for an ACL injury [18], and as a complementary measure to maximum strength aimed to follow up the recovery of muscle function after an ACLR [17]. Note that although a positive relationship between the maximum and explosive strength has been documented $[19,25,26]$, the same muscle abilities should also be partly independent $[19,20,27]$. Namely, previous studies have suggested that short-term improvements in explosive and maximum force production may require distinct training stimuli that elicit specific adaptations (e.g., high-load contractions have proved effective in increasing maximum strength, while there is equivocal evidence for its effects on explosive force production). In addition, the different phases of RFD curves can be differently modified by resistance training protocols $[14,15,19]$. Consequently, both an ACL injury and the post-ACLR rehabilitation process could selectively affect the maximum and explosive strength. This assumption has been supported by studies in which mechanisms of quadriceps weakness following an ACLR were explained by a loss of afferent feedback from the ACL $[3,28]$. The loss could have caused a prolonged disuse and hypotrophy of fast-twitch muscle fibres, which are predominantly responsible for force production in fast contractions. This could also lead to a more pronounced decrease in muscle ability to quickly exert the needed force, than in providing an overall high strength. Nevertheless, none of the cited studies have investigated time-related changes in the quadriceps and hamstrings' maximum and explosive strength during rehabilitation following an ACLR.

Another problem associated with the assessments of muscle function in individuals recovering from an ACLR is related to the use of strength measures when assessing an athlete's readiness for their return to unrestricted athletic activity. Namely, a number of studies have recommended using the side-to-side asymmetry (i.e., the ratio between the strength of the involved leg and uninvolved leg in further text "standard" asymmetries) for that purpose [5,17,29-31]. In particular, a side-to-side asymmetry below $15 \%$ has been accepted as a general criterion for athletes to return to sports activity $[4,29,32,33]$. However, an important problem with this approach could originate from the differences between legs obtained from the period following an ACLR, which could underestimate the real magnitude of asymmetry. Namely, an ACL injury typically leads to a cross-over effect in the uninvolved leg resulting in both strength and functional loss based on various central and peripheral mechanisms $[4,16,33]$. Therefore, it has recently been suggested that the use of pre-injury values could provide a more valid criterion $[4,17,30]$. Although the discussed approach has clear advantages over the standard one based on the post-ACLR side-to-side asymmetries, the pre-injury measures are usually unknown, making this approach difficult to use in routine procedures. However, in the absence of pre-injury measures, the muscle function of either both legs or only the uninvolved leg could be routinely assessed prior to the ACLR, and thereafter used for the comparison with post-ACLR measures (in further text "real" asymmetries), which could provide a potentially more valid assessment of the magnitude of side-to-side asymmetries.

To address the discussed problems, the current study was designed to evaluate the changes in the maximum and explosive strength of the quadriceps and hamstring muscle in athletes recovering from an ACLR. We hypothesized that 1) the asymmetries in explosive strength would be larger when compared with the maximum strength, as well as that 2) real asymmetries (where pre-ACLR value of the uninvolved leg was used as a control) would be larger than standard asymmetries obtained from post-ACLR measurements. The obtained results are expected to contribute to further refinement of the methods used for muscle function testing in individuals recovering after an ACLR.

\section{Methods}

\subsection{Participants}

According to standard guidelines [34] with effect size of 0.5, power of 0.8 and an alpha level of 0.05 (calculated by $\mathrm{G}^{*}$ Power 3.1 free software [35]), the required sample size was 15. Twenty-three ACLR participants were initially recruited through the Clinic for Orthopaedic Surgery and Traumatology, but three were lost to follow-up. The remaining 20 participants were males soccer $(\mathrm{N}=12)$, handball (5) and judo (3) competitors engaged in professional sport at the national level. Their age was $22 \pm 0.9$ years, body mass $84.0 \pm 2.5 \mathrm{~kg}$, height $180.3 \pm 0.9 \mathrm{~cm}$ (data presented as mean $\pm \mathrm{SE}$ ). The inclusion criteria were: first ACL injury and participation in competitive sports at the national level or higher. The exclusion criteria were: other knee ligaments injured, history of concurrent fractures, osteoarthritis, as well as hereditary and neuromuscular diseases. The ACLR procedure was performed by the same experienced surgeon, using the bone-patellar-bone tendon (BPTB) autograft. Following the surgery, the participants were allocated to a standard postoperative rehabilitation program for athletes. All participants received a complete explanation regarding the purpose and procedures of the study, as well as the possible risks. Ethical clearance for the study was obtained from the pertinent institutional review board. In line with the Helsinki Declaration, the institutionally approved informed consents were obtained from participants and their rights were protected.

\subsection{Testing procedure}

All measurements were taken from three separate sessions: pre-ACLR (i.e. within seven days prior to surgery), as well as four and six months post-ACLR. Standard clinical assessments included questionnaires (International Knee Documentation Committee (IKDC) subjective score [36] and Tegner score [37]) and knee laxity test performed with KT1000 instrumented arthrometer (MEDmetric Corporation, San Diego, CA) at $13.61 \mathrm{~kg}$ of force [17]. All clinical assessments were performed by an experienced orthopedist. At six months postACLR, all participants performed a one-leg hop test for distance as a functional assessment of the dynamic stability of the knee. The hop test was performed both with the uninvolved leg and involved leg, according to the standard procedure [38].

Strength measurements were performed within a university research laboratory, using a Kin-Com AP125 isokinetic dynamometer (Chatex Corp., Chattanooga, Tennessee, USA) set to isometric conditions. The subjects were seated in an upright position and fixed to the testing apparatus, with the straps around the pelvis, the thigh, and malleoli. The axis of rotation of the dynamometer was aligned with the lateral femoral epicondyle. The knee angle was fixed at $45^{\circ}$ in flexion ( $0^{\circ}$ corresponded to full extension) $[39,40]$. Prior to muscle strength testing, each subject was given a five min warm-up period on a stationary bicycle, followed by passive stretching exercises, and two submaximum isometric contraction trials performed using the dynamometer. The uninvolved leg was always tested first. A real time visual feedback of the force-time curve was available during the assessment of the maximum isometric strength $[19,41]$. The same experienced test leader supervised all the tests. A detailed explanation and a qualified demonstration were both provided prior to each muscle 
function test, and standardized verbal encouragement was used. Depending on the muscle group tested, subjects were instructed either to extend or flex the knee "as fast and as hard as possible" aimed to provide the indices of both the maximum and explosive strength $[19,42$, 43]. Two trials per each muscle were performed. Each contraction was sustained for five s and the rest period between them was $60 \mathrm{~s}$. Trials with visible initial countermovement were repeated $[18,19]$.

\subsection{Data acquisition and processing}

A custom-made Lab View application (National Instruments Corporation, Austin, TX, USA) was used for data acquisition and processing. Force-time signal was sampled at $500 \mathrm{~Hz}$ and low-pass filtered $(10 \mathrm{~Hz})$ using a fourth-order (zero-phase lag) Butterworth filter. The onset of the contraction was defined as the point in time where the first derivative of the force-time curve exceeded the baseline by $3 \%$ of its maximum value. The maximum strength was assessed through the maximum muscle force $\left(F_{\max }\right)$. The explosive strength was assessed through four variables derived from the slope of the force-time curves. Specifically, $\mathrm{RFD}_{\text {max }}$ represented the maximum slope of the force-time curve, while $\mathrm{RFD}_{50 \mathrm{~ms}}, \mathrm{RFD}_{150 \mathrm{~ms}}$ and $\mathrm{RFD}_{250 \mathrm{~ms}}$ represented the average slopes over time intervals 0 to $50 \mathrm{~ms}$ (early phase of strength development), 0 to $150 \mathrm{~ms}$, and 0 to $250 \mathrm{~ms}$ (late phase of strength development) relative to the onset of contraction according to the method previously described by Aagaard et al. [13]. RFD during the early phase of muscle contraction $(<100 \mathrm{~ms})$ should represent influence of neural drive and intrinsic contractile properties of the muscle, whereas RFD during later time intervals (i.e. $>100 \mathrm{~ms}$ ) should reflect influence of maximum muscle strength $[15,19]$. To control for the possible confounding effects of body size, all strength variables were normalized with respect to the body mass on power $2 / 3$ [44-46].

Side-to-side asymmetries between the uninvolved leg and the involved leg were calculated in two ways. First, they were calculated as asymmetries for each session (i.e., pre-ACLR, four and six months post-ACLR) as (UNINVOLVED - INVOLVED) / UNINVOLVED $\times 100$ (standard asymmetries). Second, they were also calculated for the sessions conducted four and six months post-ACLR with respect to the data obtained pre-ACLR as (UNINVOLVED preACLR $_{-}$INVOLVED $\left._{\text {postACLR }}\right)$ / UNINVOLVED preACLR $\times 100$ (real asymmetries).

\subsection{Statistical analysis}

Descriptive statistics (mean and standard errors) were calculated for all variables. Changes in the mean IKDC, Tegner, and KT1000 scores were evaluated applying ANOVA with repeated measures (obtained pre-, four and six months post-ACLR). Mixed model ANOVA [factors being 'leg' and 'session' (repeated factor)] was used to evaluate the differences in the $\mathrm{F}_{\max }$ and RFD values both between the legs (uninvolved vs. involved), as well as among three sessions (pre-ACLR vs. four and six months post-ACLR and 4 months vs. 6 months post-ACLR). Where significant main effects and their interactions were found, the Bonferroni post-hoc test was applied [p values were SPSS Bonferroniadjusted for the purpose of comparison with an assumed family-wise alpha level of $0.05(0.01)]$. In case of significant main effects without interactions, separate simple ANOVAs (factor 'leg') were applied to explore between-leg differences within each session. In addition, to explore the differences across the sessions within each leg, simple ANOVA's for each leg (factor 'session') were performed [47].

To test the first hypothesis, $5 \times 3$ mixed model ANOVA (main factors being 'variable' and 'session') was used to compare the limb standard asymmetries between the $\mathrm{F}_{\max }$ and RFD variables obtained from particular sessions. Where significant main effects and their interactions were found, the Bonferroni post-hoc test was applied. In case of significant main effects without interactions, simple ANOVAs (factor 'variable') across variables at pre-ACLR, 4 months and 6 months post-ACLR were performed. In addition, simple ANOVA (factor 'session') were applied to compare the time related changes in each variable. In case of significant factors the Bonferroni post-hoc test was applied. To compare real asymmetries, simple ANOVA (factor 'variable') was performed separately at 4 and 6 months post-ACLR. Where significant main effects and their interactions were found, the Bonferroni post-hoc test was applied.

To test the second hypothesis, paired-sample T-test was used to determine the differences between the standard and real asymmetries, four and six months post-ACLR. Finally, a one-group T-test was used to compare standard and real asymmetries with the criterion being a safe return to sport (i.e., the asymmetry below 15\%). All analyses were performed separately for quadriceps and hamstrings. The level of statistical significance was set to $p=0.05$. Data were analyzed using SPSS 20.0 software (SPSS Inc. Chicago, IL, USA).

\section{Results}

Outcomes of the clinical tests are shown in Table 1. Note that the data suggest that the normal values were reached six months post-ACLR. Regarding the results of the one leg hop test, the distances reached by the involved leg and uninvolved leg, respectively, were $164 \pm 25 \mathrm{~cm}$ and $184 \pm 22 \mathrm{~cm}(\mathrm{t}=5.5 ; \mathrm{p}<0.01)$. The asymmetry calculated from the data was $10.9 \pm 8.7 \%$

Representative quadriceps and hamstrings' force-time profiles obtained from a representative subject for the involved leg (dashed line) and uninvolved (solid line) leg are shown in Fig. 1. The data suggest lower $\mathrm{F}_{\max }$ and RFD values of the involved leg across all sessions.

Descriptive data for the strength and explosive strength variables (i.e., $F_{\max }$ and various indices of RFD) of quadriceps are presented in Table 2. When the differences were tested, the main factor of 'leg' was significant ( $F$ ranging from 30.7 to 69.6; all $\mathrm{p}<0.01$ ) resulting in lower values of $\mathrm{F}_{\max }$ and RFD measures of the involved leg than of the uninvolved leg. The main factor of 'time' was also significant ( $F$ ranging from 4.09 to 6.06; all $\mathrm{p}<0.05$ ), mainly due to lower values obtained 4 months post-ACLR than both pre-ACLR and 6 months post-ACLR. Step-down analysis by simple ANOVAs revealed significant differences between the involved leg and the uninvolved leg within each session (all $\mathrm{p}<0.01$ ). In addition, $\mathrm{F}_{\max }$ and RFD of the involved leg were lower at 4 months post-ACLR $(19-27 \%)$ than both at pre-ACLR (all p < 0.05) and 6 months post-ACLR (17-22\%). Change in the uninvolved leg was not significant, being up to $10 \%$ across the sessions. Regarding the hamstrings (Table 3), only the main factor of 'leg' was significant $(\mathrm{F}=10.370-16.471$; all $\mathrm{p}<0.05)$, resulting in lower values of $\mathrm{F}_{\max }$ and RFD measures of the involved leg than that of the uninvolved leg. Although factor 'session' was not significant, hamstrings' RFD measures of the involved side were approximately $17 \%$ lower at 4 months post-ACLR than at pre-ACLR, while the same change in $\mathrm{F}_{\max }$ was only $5 \%$.

Standard asymmetries in the $\mathrm{F}_{\max }$ and RFD variables are depicted in Fig. 2. Mixed model ANOVA applied on quadriceps revealed significant main effects of 'variable' $(\mathrm{F}=15.457 ; \mathrm{p}<0.01)$ and 'session' $(\mathrm{F}=9.417 ; \mathrm{p}<0.01)$ but not of interaction. Of particular importance for the first hypothesis are larger asymmetries in the explosive than in the maximum strength. Specifically, when simple ANOVAs were applied within each of the sessions, post-hoc indicated that the asymmetries for $\mathrm{RFD}_{50}, \mathrm{RFD}_{150}$ and $\mathrm{RFD}_{250}$ were larger than for $\mathrm{F}_{\max }$ both pre-ACLR $(\mathrm{F}=8.354 ; \mathrm{p}<0.01)$ and 4 months post-ACLR $(\mathrm{F}=8.318 ; \mathrm{p}<0.01)$, but not 6 months post-ACLR. Furthermore, all asymmetries observed 4 months post-ACLR were larger than both pre- and 6 months post-ACLR ( $p<0.01$ ). Regarding hamstrings, only the main effect of 'session' was significant $(\mathrm{F}=8.747 ; \mathrm{p}<0.05)$, due to larger asymmetries observed 4 months post-ACLR than both pre-ACLR and 6 months post-ACLR. When real asymmetries were analyzed, main effect of 'variable' was significant for quadriceps asymmetries, both at 4 months $(\mathrm{F}=11.554 ; \mathrm{p}<0.01)$ and 6 months post-ACLR $(\mathrm{F}=20.856 ; \mathrm{p}<0.01)$. The post-hoc test revealed that the asymmetries in RFD were larger than in $\mathrm{F}_{\max }$ at both 4 and 6 months post-ACLR (Fig. 3; left panel).

To test the second hypothesis of the study, we compared the standard and real asymmetries (Fig. 3). At 4 months post-ACLR (upper panel), there were no differences between the real and standard asymmetries in both of the muscle groups. However, real asymmetries observed 6 months post-ACLR were larger than the standard asymmetries

Table 1

Descriptive statistics for clinical scores.

\begin{tabular}{lccc}
\hline & Pre-ACLR & 4 months post-ACLR & 6 months post-ACLR \\
\hline IKDC & $69.1 \pm 2.4$ & $76.6 \pm 2.3^{\dagger}$ & $83.5 \pm 1.6^{\dagger}$ \\
Lysholm & $76.1 \pm 3.3$ & $84.6 \pm 3.6^{\dagger}$ & $89.4 \pm 2.8$ \\
Activity & $7.4 \pm 0.5$ & $5.2 \pm 0.4^{\ddagger}$ & $6.5 \pm 0.4^{\ddagger}$ \\
KT1000 & $6.3 \pm 0.5$ & $1.7 \pm 0.3^{\ddagger}$ & $2.1 \pm 0.3^{\dagger}$ \\
\hline
\end{tabular}

All data are presented as mean $\pm \mathrm{SE}$.

+ Significantly different from previous session $(\mathrm{p}<0.05)$

* Significantly different from previous session $(\mathrm{p}<0.01)$. 

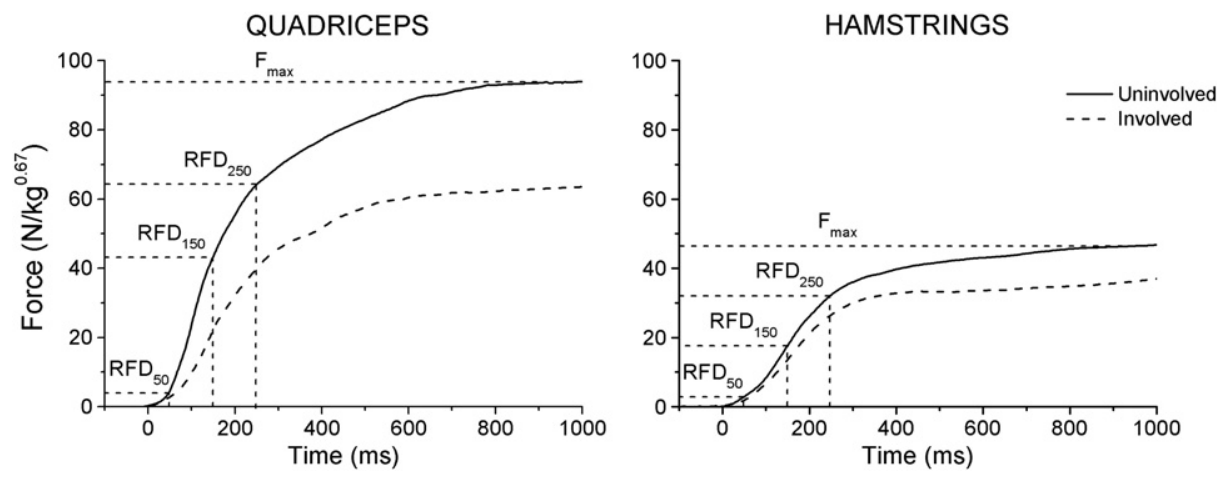

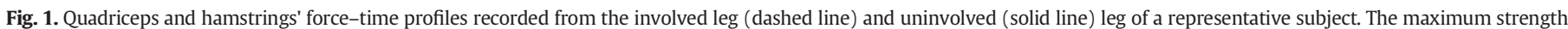

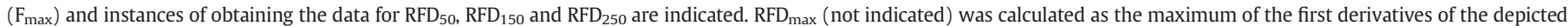
force-time curves.

for quadriceps (see lower panel of Fig. 3). In particular, the differences were significant for $\mathrm{RFD}_{\max }, \mathrm{RFD}_{50}$ and $\mathrm{RFD}_{150}$ ( $T$-test range 2.131-3.082; all $\mathrm{p}<0.05$ ) but not for the $\mathrm{F}_{\max }$. Regarding hamstrings, no differences between the real and the standard asymmetries were observed. It should be noted that both the standard and real quadriceps asymmetries observed 6 months post-ACLR were significantly larger than the generally accepted criterion for a safe return to unrestricted activity (one sample $T$-test range from 3.44 to 8.86; $\mathrm{p}<0.01$ ), particularly in explosive strength sports.

\section{Discussion}

In the present study we tested the quadriceps and hamstring muscle in athletes recovering from an ACLR and evaluated the asymmetries in their maximum and explosive strength. Regarding the first hypothesis, we found that the asymmetries in the indices of explosive strength were larger than the asymmetries in the maximum strength throughout all measurements. Regarding the second hypothesis, we found that the asymmetries in indices of explosive strength were even more prominent when the pre-ACLR value (i.e., real) of the uninvolved leg was used as a control. Finally, we also showed that the asymmetries in the quadriceps explosive and maximum strength 6 months post-ACLR were still above the upper limit that is considered safe for a return to strenuous activities.

As expected, the maximum strength only revealed significant time-related changes in the involved quadriceps. The lowest strength values were recorded 4 months post-ACL, whereas at 6 months

Table 2

Descriptive statistics for quadriceps' data.

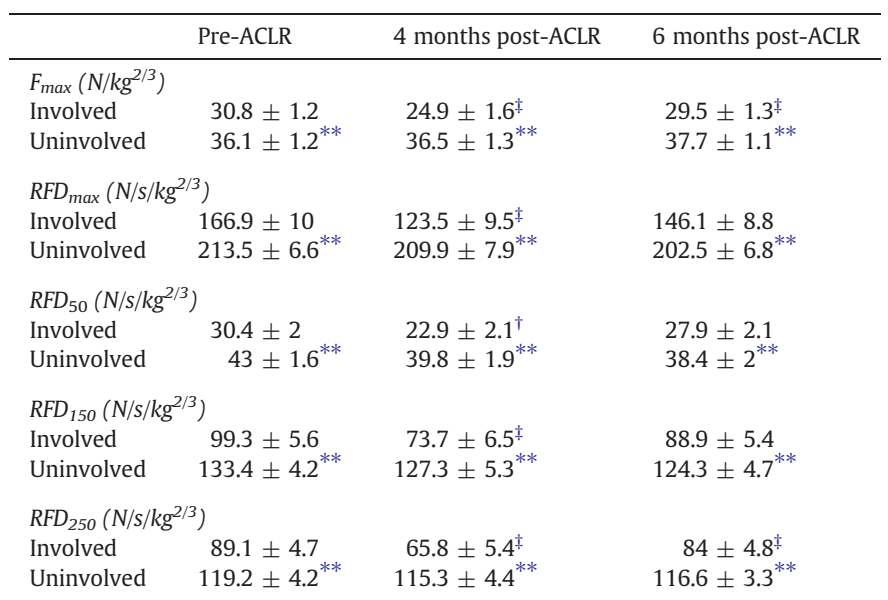

All data are presented as mean \pm SE; $p$ values were SPSS adjusted (Bonferroni) so they could be compared to an assumed family-wise alpha level of 0.05 (0.01).

** Significantly different from the involved leg $(\mathrm{p}<0.01)$.

$\dagger$ Significantly different from previous session $(\mathrm{p}<0.05)$.

* Significantly different from previous session $(\mathrm{p}<0.01)$.
post-ACLR the maximum strength nearly recovered to the pre-ACLR level. These findings are generally in line with the previous studies $[7,32,48]$. The loss in quadriceps explosive strength was accompanied by a minor reduction of explosive strength in the hamstrings of the involved leg, as well as in the quadriceps of the uninvolved leg. At 6 months post-ACLR, the indices of explosive strength of the involved quadriceps were up to $14 \%$ lower than the corresponding pre-ACLR values.

The most important findings of the present study could be those based on the comparison of the asymmetries in the maximum and explosive strength. The asymmetries in the quadriceps explosive strength (both early and late RFD) were already more prominent than in asymmetries in the maximum strength during the pre-ACLR measurement, and also larger at both post-ACLR measurements. The asymmetries recorded 6 months post-ACLR decreased to values that were similar to the values observed pre-ACLR, but still remained above (i.e. $22 \%$ for $\mathrm{F}_{\max }$ and $26-28 \%$ RFD measures) the criterion for athletes to return to competitive sports activity (i.e., $15 \%$; $[4,17,31]$ ). It should be noted that these asymmetries were even larger when the corresponding pre-ACLR measures of the uninvolved leg were used as a control, being up to $39 \%$ of the explosive strength. A large discrepancy in the size of the asymmetry and rate of recovery between the maximum and explosive strength could indicate that the ability of rapid force production could be an independent strength quality [19,20,27]. However, similar changes observed in both early and late RFD suggests that an ACLR affects both the neural and contractile properties of the involved leg. Namely, the underlying mechanism of persistent

Table 3

Descriptive statistics for hamstrings' data.

\begin{tabular}{|c|c|c|c|}
\hline & Pre-ACLR & 4 months post-ACLR & 6 months post-ACLR \\
\hline \multicolumn{4}{|l|}{$F_{\max }\left(\mathrm{N} / \mathrm{kg}^{2 / 3}\right)$} \\
\hline Involved & $15.2 \pm 0.8$ & $14.5 \pm 0.8$ & $15.8 \pm 0.5$ \\
\hline Uninvolved & $18.0 \pm 0.8$ & $18.2 \pm 0.8$ & $18.2 \pm 0.5$ \\
\hline \multicolumn{4}{|c|}{$R F D_{\max }\left(\mathrm{N} / \mathrm{s} / \mathrm{kg}^{2 / 3}\right)$} \\
\hline Involved & $86.0 \pm 4.4$ & $70.6 \pm 3.7$ & $81.2 \pm 2.9$ \\
\hline Uninvolved & $91.7 \pm 4.1$ & $92.1 \pm 4.3$ & $90.9 \pm 3.3$ \\
\hline \multicolumn{4}{|c|}{$R F D_{50}\left(\mathrm{~N} / \mathrm{s} / \mathrm{kg}^{2 / 3}\right)$} \\
\hline Involved & $14.5 \pm 1.0$ & $11.8 \pm 0.8$ & $13.8 \pm 0.7$ \\
\hline Uninvolved & $16.1 \pm 1.0$ & $16.2 \pm 0.9$ & $16.4 \pm 0.8$ \\
\hline \multicolumn{4}{|c|}{$R F D_{150}\left(\mathrm{~N} / \mathrm{s} / \mathrm{kg}^{2 / 3}\right)$} \\
\hline Involved & $47.3 \pm 2.9$ & $39.2 \pm 2.6$ & $45.9 \pm 2.3$ \\
\hline Uninvolved & $53.3 \pm 2.6$ & $53.3 \pm 2.8$ & $53.4 \pm 2.1$ \\
\hline \multicolumn{4}{|c|}{$R F D_{250}\left(\mathrm{~N} / \mathrm{s} / \mathrm{kg}^{2 / 3}\right)$} \\
\hline Involved & $50.5 \pm 2.5$ & $43.2 \pm 2.3$ & $50.0 \pm 1.8$ \\
\hline Uninvolved & $57.3 \pm 2.4$ & $57.0 \pm 2.3$ & $57.4 \pm 1.8$ \\
\hline
\end{tabular}

All data are presented as mean \pm SE. 
QUADRICEPS

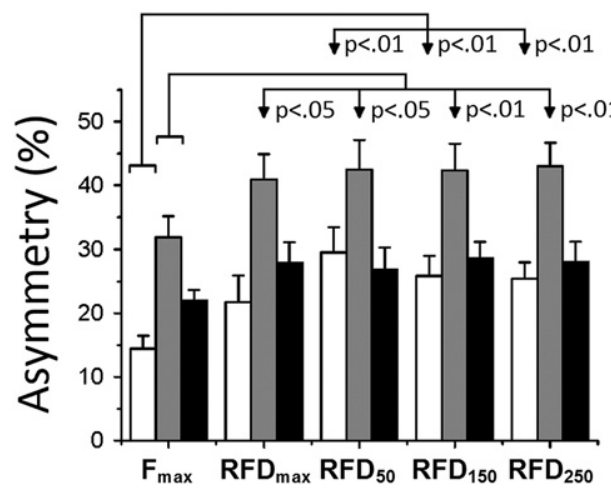

HAMSTRINGS

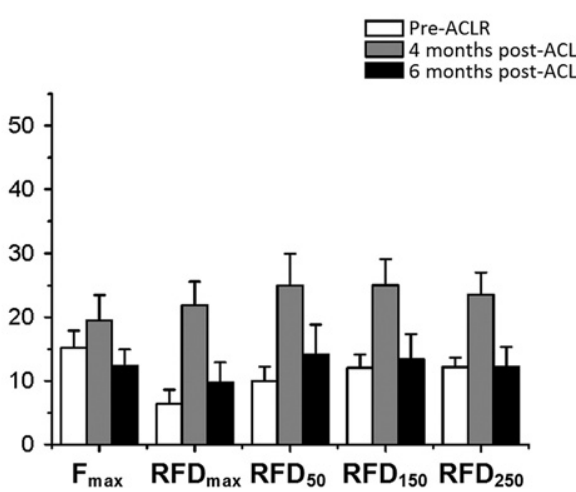

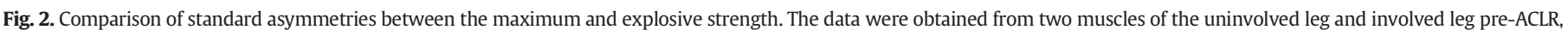

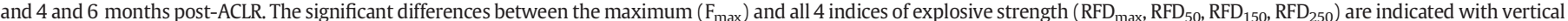
arrows.

quadriceps weakness often observed within the first 6 months of an ACLR could be the loss of afferent feedback from the ACL that can contribute to a gamma loop dysfunction, along with the impaired neuromuscular activation particularly pronounced in the fast twitch motor units [2,3]. Thus, a profound muscle weakness typically associated with the ACL injury and reconstruction could be particularly pronounced regarding the explosive strength. As a result, one could generally conclude that in addition to maximum strength, the indices of explosive strength (both early and late RFD) should be routinely obtained for the assessment of the quadriceps and hamstring muscle function following an ACLR. This could be of particular importance for both clinicians and conditioning specialists since the inclusion of both measures in the follow-up of the patients' recovery could provide additional information regarding the underlying impairments in the neural and/or contractile mechanisms.

\section{QUADRICEPS 4 months}

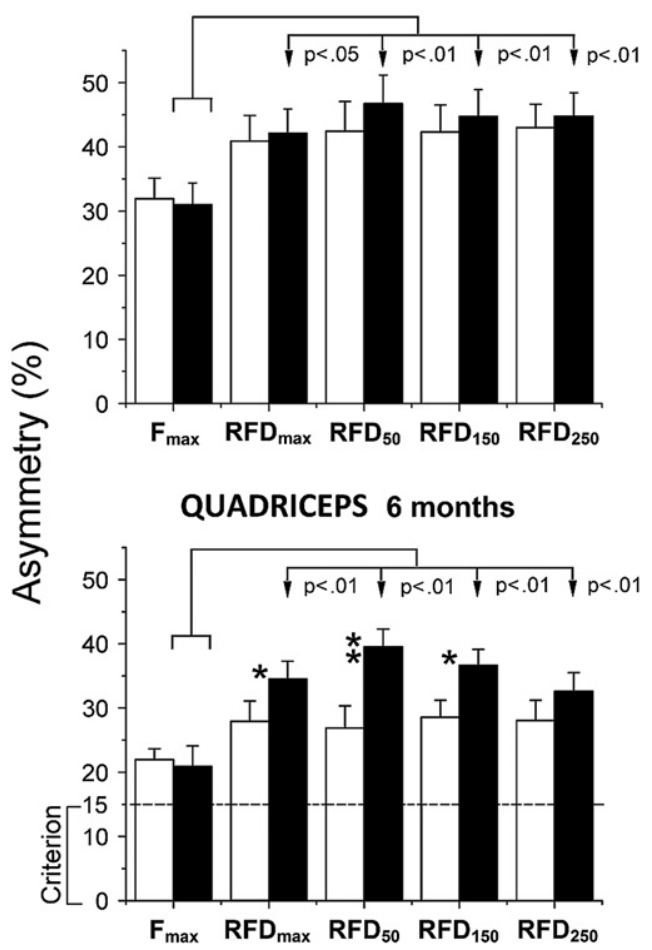

Another set of potentially important findings is related to our second hypothesis that the asymmetries calculated relative to pre-ACLR value of the uninvolved leg (real asymmetries) would be larger than the standard post-ACLR asymmetries. Traditionally, the changes in muscle function following an ACLR have been evaluated through the side-to-side asymmetries derived from measurements taken at a particular time post-ACLR (i.e., standard asymmetries). The underlying assumption has been that the legs were equal regarding the muscle size and strength prior to injury, while the properties of the uninvolved leg do not change over the rehabilitation period. However, this approach has been criticized since strength of the uninvolved side often decreases over time due to the cross-over inhibition of motor activation, as well as due to either de-conditioning or insufficient reconditioning $[2,29,33,49]$. This concept is clearly supported by our findings where standard asymmetries in the quadriceps explosive

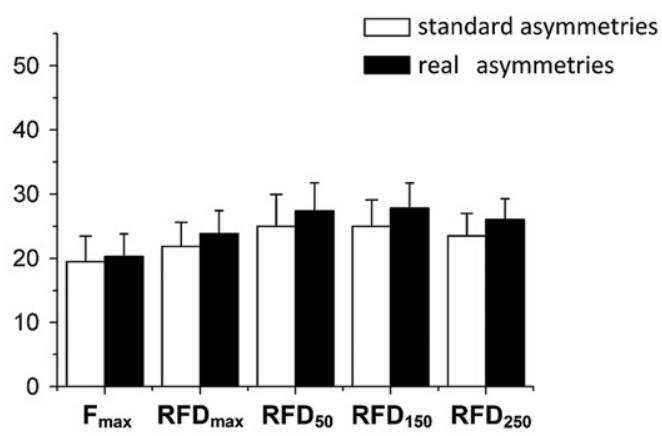

HAMSTRINGS 6 months

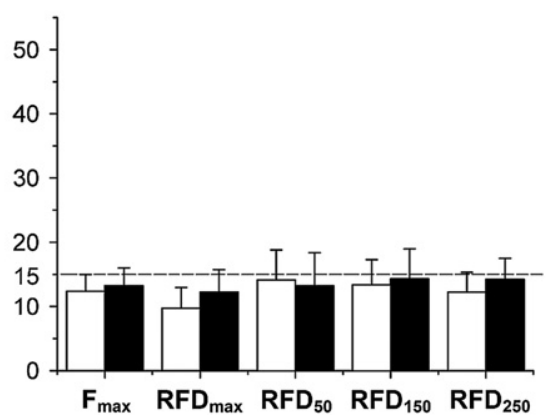

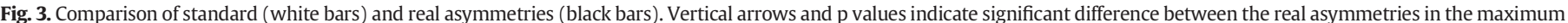

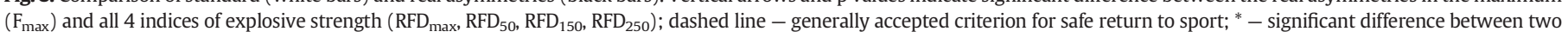
types of asymmetries, $\mathrm{p}<0.05$; $^{* *}-$ significant difference between two types of asymmetries, $\mathrm{p}<0.01$. 
strength were significantly lower than the corresponding real asymmetries. This could be of particular importance for practice since reliance on post-ACLR measures could lead to underestimation of the real asymmetries in muscle function, therefore concealing deficits that may limit athletes' potentials for a safe return to competitive sports. A recent review reported that, although the great majority (i.e., nearly $90 \%$ ) of athletes reached the criterion considered for normal or near-normal standard asymmetries (i.e., below 15\%), the return to sport still remained low [50]. This suggests that the criterion based on standard asymmetries used in the decision-making process regarding recovery and readiness for return to sports may not be demanding enough.

Our results indicate that the athletes recovering following ACLR could benefit from resistance training designed to improve not only the maximum, but the explosive strength as well. Previous studies have shown that a combination of high-force low-velocity, low-force high-velocity, and high-force high-velocity exercises could elicit substantial enhancements in explosive strength $[13,15,17]$. Furthermore, a training for neural adaptations (i.e. aimed to activate the musculature as rapidly as possible) appears to be an important training strategy to enhance RFD, as it is necessary to invoke rapid motor-unit activation [15,42]. Finally, as shown by Gruber and Gollhofer [51], the sensorimotor training, often applied in rehabilitation of injured athletes, could be able to enhance the strength capacity at the onset of maximum voluntary contraction, which is characterized by higher early neural muscle activation and, consequently, increased RFD. However, further studies aimed to explore the potential benefit of training strategies designed to improve explosive strength in individuals rehabilitating following ACLR are necessary.

Although our study provided novel data regarding the importance for the inclusion of explosive strength measures in follow-ups of athletes recovering from an ACLR, further research is apparently needed to address some important limitations of the present study. First, the selected sample was heterogeneous regarding the athletic background, while the sample size was relatively limited not only in number, but also in the choice. Second, the lack of female subjects could also be a limiting factor, particularly considering a higher incidence of ACL injuries in female athletes. Third, the applied pre-ACLR strength exercise program was not controlled to prevent its potentially confounding effects on the observed outcomes. Fourth, use of single-joint isometric contractions could be of questionable ecological validity, since measuring the forces at attempted single joint movements is not specific to performance of most human activities. Additionally, the underlying neural activation pattern of isometric tests could be different from the same pattern at high velocity multi-joint movements that during which ACL typically occur. However, note also that any attempt to routinely obtain the indices of maximum and explosive strength from complex multi-joint movements inevitably imposes numerous methodological problems, such as those related to standardization, coordination, and skill. Finally, although our study revealed similar asymmetries in all indices of explosive strength, the underlying mechanisms of persistent quadriceps weakness caused by an ACLR in terms of neural and contractile properties still partly remain unknown.

\section{Conclusions}

Evaluation of muscle function pre- and post-ACLR revealed larger asymmetries in explosive than in maximum strength, while the asymmetries in strength measures were even more prominent when the pre-ACLR value of the uninvolved leg was used as a control. Therefore, we conclude that (1) in addition to maximum strength, indices of explosive strength should be included in monitoring recovery of muscle function following an ACLR, while (2) pre-injury/reconstruction values should be used for the post-ACLR side-to-side comparisons, providing a more valid criterion regarding the muscles' recovery and readiness for a return to sports. In general, the obtained finding could contribute to further refinement of the methods applied to monitoring the rehabilitation process and recovery following an ACLR.

\section{Conflict of interest}

The authors of this manuscript have no conflict of interest to declare.

\section{Acknowledgment}

Support for this study was provided by the National Institute of Health (R21AR06065) and the Serbian Ministry of Education, Science and Technological Development (\#175037; and \#175012). We thank Dr. Darko Milovanovic for clinical evaluation of the participants. Sponsors had no role in the study design; in the collection, analysis and interpretation of data; in the writing of the manuscript; and in the decision to submit the manuscript for publication.

\section{References}

[1] Williams GN, Buchanan TS, Barrance PJ, Axe MJ, Snyder-Mackler L. Quadriceps weakness, atrophy, and activation failure in predicted noncopers after anterior cruciate ligament injury. Am J Sports Med 2005;33:402-7.

[2] Konishi Y, Aihara Y, Sakai M, Ogawa G, Fukubayashi T. Gamma loop dysfunction in the quadriceps femoris of patients who underwent anterior cruciate ligament reconstruction remains bilaterally. Scand J Med Sci Sports 2007;17:393-9.

[3] Konishi Y, Fukubayashi T, Takeshita D. Mechanism of quadriceps femoris muscle weakness in patients with anterior cruciate ligament reconstruction. Scand J Med Sci Sports 2002;12:371-5

[4] Thomee R, Neeter C, Gustavsson A, Thomee P, Augustsson J, Eriksson B, et al Variability in leg muscle power and hop performance after anterior cruciate ligament reconstruction. Knee Surg Sports Traumatol Arthrosc 2012;20:1143-51.

[5] Adams D, Logerstedt DS, Hunter-Giordano A, Axe MJ, Snyder-Mackler L. Current concepts for anterior cruciate ligament reconstruction: a criterion-based rehabilitation progression. J Orthop Sports Phys Ther 2012;42:601-14.

[6] Hartigan EH, Zeni Jr J, Di Stasi S, Axe MJ, Snyder-Mackler L. Preoperative predictors for noncopers to pass return to sports criteria after ACL reconstruction. J Appl Biomech 2012;28:366-73.

[7] Moisala AS, Jarvela T, Kannus P, Jarvinen M. Muscle strength evaluations after ACL reconstruction. Int J Sports Med 2007;28:868-72.

[8] Lord JP, Aitkens SG, McCrory MA, Bernauer EM. Isometric and isokinetic measurement of hamstring and quadriceps strength. Arch Phys Med Rehabil 1992; 73:324-30.

[9] Reinking MF, Bockrath-Pugliese K, Worrell T, Kegerreis RL, Miller-Sayers K, Farr J Assessment of quadriceps muscle performance by hand-held, isometric, and isokinetic dynamometry in patients with knee dysfunction. J Orthop Sports Phys Ther 1996;24:154-9.

[10] Morrissey MC, Hooper DM, Drechsler WI, Hill HJ. Relationship of leg muscle strength and knee function in the early period after anterior cruciate ligament reconstruction. Scand J Med Sci Sports 2004;14:360-6.

[11] Jaric S. Muscle strength testing: use of normalisation for body size. Sports Med 2002; 32:615-31.

[12] Wilson GJ, Murphy AJ. The use of isometric tests of muscular function in athletic assessment. Sports Med 1996;22:19-37.

[13] Aagaard P, Simonsen EB, Andersen JL, Magnusson P, Dyhre-Poulsen P. Increased rate of force development and neural drive of human skeletal muscle following resistance training. J Appl Physiol 2002;93:1318-26.

[14] Tillin NA, Pain MTG, Folland J. Explosive force production during isometric squats correlates with athletic performance in rugby union players. J Sports Sci 2013; 31:66-76.

[15] Oliveira FB, Oliveira AS, Rizatto GF, Denadai BS. Resistance training for explosive and maximal strength: effects on early and late rate of force development. J Sports Sci Med 2013;12:402-8.

[16] Folland JP, Buckthorpe MW, Hannah R. Human capacity for explosive force production: neural and contractile determinants. Scand J Med Sci Sports 2013 [first published online].

[17] Angelozzi M, Madama M, Corsica C, Calvisi V, Properzi G, McCaw ST, et al. Rate of force development as an adjunctive outcome measure for return-to-sport decisions after anterior cruciate ligament reconstruction. J Orthop Sports Phys Ther 2012; 42:772-80.

[18] Zebis MK, Andersen LL, Ellingsgaard H, Aagaard P. Rapid hamstring/quadriceps force capacity in male vs. female elite soccer players. J Strength Cond Res 2011; 25:1989-93.

[19] Andersen LL, Aagaard P. Influence of maximal muscle strength and intrinsic muscle contractile properties on contractile rate of force development. Eur J Appl Physiol 2006;96:46-52.

[20] Mirkov DM, Nedeljkovic A, Milanovic S, Jaric S. Muscle strength testing: evaluation of tests of explosive force production. Eur J Appl Physiol 2004;91:147-54.

[21] Paasuke M, Ereline J, Gapeyeva H. Knee extension strength and vertical jumping performance in Nordic combined athletes. J Sports Med Phys Fitness 2001; 41:354-61. 
[22] Izquierdo M, Aguado X, Gonzalez R, Lopez J, Häkkinen K. Maximal and explosive force production capacity and balance performance in men of different ages. Eur J Appl Physiol 1999;79:260-7.

[23] Pijnappels M, Reeves ND, van Dieën JH. Identification of elderly fallers by muscle strength measures. Eur J Appl Physiol 2008;102:585-92.

[24] Maffiuletti NA, Bizzini M, Widler K, Munzinger U. Asymmetry in quadriceps rate of force development as a functional outcome measure in TKA. Clin Orthop Relat Res 2010;468:191-8.

[25] Prebeg G, Cuk I, Suzovic D, Stojiljkovic S, Mitic D, Jaric S. Relationships among the muscle strength properties as assessed through various tests and variables. J Electromyogr Kinesiol 2013;23:455-61.

[26] Ryushi T, Hakkinen K, Kauhanen H, Komi P. Muscle fiber characteristics, muscle cross-sectional area and force production in strength athletes, physically active males and females. Scand J Sports Sci 1988;10:7-15.

[27] Mcguigan MR, Newton MJ, Winchester JB, Nelson AG. Relationship between isometric and dynamic strength in recreationally trained men. J Strength Cond Res 2010;24:2570-3

[28] Konishi Y, Fukubayashi T, Takeshita D. Possible mechanism of quadriceps femoris weakness in patients with ruptured anterior cruciate ligament. Med Sci Sports Exerc 2002;34:1414-8

[29] Engelen-van Melick N, van Cingel RE, Tijssen MP, Nijhuis-van der Sanden MW. Assessment of functional performance after anterior cruciate ligament reconstruction: a systematic review of measurement procedures. Knee Surg Sports Traumatol Arthrosc 2013;21:869-79.

[30] Keays SL, Bullock-Saxton J, Keays AC, Newcombe P. Muscle strength and function before and after anterior cruciate ligament reconstruction using semitendonosus and gracilis. Knee 2001;8:229-34.

[31] Knezevic OM, Mirkov DM. Strength assessment in athletes following an anterior cruciate ligament injury. Kinesiology 2013:45:3-15.

[32] Ageberg E, Roos HP, Silbernagel KG, Thomee R, Roos EM. Knee extension and flexion muscle power after anterior cruciate ligament reconstruction with patellar tendon graft or hamstring tendons graft: a cross-sectional comparison 3 years post surgery. Knee Surg Sports Traumatol Arthrosc 2009;17:162-9.

[33] Hiemstra LA, Webber S, MacDonald PB, Kriellaars DJ. Contralateral limb strength deficits after anterior cruciate ligament reconstruction using a hamstring tendon graft. Clin Biomech (Bristol, Avon) 2007;22:543-50.

[34] Cohen J. Statistical power analysis for the behavioral sciences, 567(20-27). Hillsdale, NJ: Lawrence Erlbaum Associates; 1988.

[35] Faul F, Erdfelder E, Buchner A, Lang AG. Statistical power analyses using G*Power 3.1: tests for correlation and regression analyses. Behav Res Methods 2009; 41:1149-60.

[36] Hefti F, Muller W, Jakob RP, Staubli HU. Evaluation of knee ligament injuries with the IKDC form. Knee Surg Sports Traumatol Arthrosc 1993;1:226-34.
[37] Tegner Y, Lysholm J, Lysholm M, Gillquist J. A performance test to monitor rehabilitation and evaluate anterior cruciate ligament injuries. Am J Sports Med 1986; $14: 156-9$.

[38] Fitzgerald GK, Lephart SM, Hwang JH, Wainner RS. Hop tests as predictors of dynamic knee stability. J Orthop Sports Phys Ther 2001;31:588-97.

[39] Zemach L, Almoznino Y, Barak Y, Dvir Z. Quadriceps insufficiency in patients with knee compromise: how many velocities should an isokinetic test protocol consist of? Isokinet Exerc Sci 2009;17:129-33.

[40] Knezevic O, Mirkov D, Milovanovic D, Kadija M, Jaric S. Alternating consecutive maximum contractions as a test of muscle function in athletes following ACL reconstruction. J Hum Kinet 2012;35:5-13.

[41] Knezevic OM, Mirkov DM, Milovanovic D, Kadija M, Jaric S. Evaluation of isokinetic and isometric strength measures for monitoring muscle function recovery following ACL reconstruction. J Strength Cond Res 2013.

[42] Sahaly R, Vandewalle $H$, Driss T, Monod $H$. Maximal voluntary force and rate of force development in humans - importance of instruction. Eur J Appl Physiol 2001; 85:345-50.

[43] Bemben MG, Clasey JL, Massey BH. The effect of the rate of muscle contraction on the force-time curve parameters of male and female subjects. Res Q Exerc Sport 1990; 61:96-9.

[44] Astrand P, Rodahl K. Textbook of work physiology: physiological bases of exercise; 1986 [86]

[45] Jaric S. Role of body size in the relation between muscle strength and movement performance. Exerc Sport Sci Rev 2003;31:8-12.

[46] Marković G, Mirkov D, Jarić S. Maximum exercise performance and body size. In: Swan RL, editor. Trends in exercise and health research. Hauppauge, NY: Nova Science Publishers, Inc.; 2005

[47] Vincent WJ. Statistics in kinesiology, 3rd ed., 312(210-211); 2005.

[48] Lautamies R, Harilainen A, Kettunen J, Sandelin J, Kujala UM. Isokinetic quadriceps and hamstring muscle strength and knee function 5 years after anterior cruciate ligament reconstruction: comparison between bone-patellar tendon-bone and hamstring tendon autografts. Knee Surg Sports Traumatol Arthrosc 2008; 16:1009-16.

[49] Strandberg S, Lindström M, Wretling M-L, Aspelin P, Shalabi A. Muscle morphometric effect of anterior cruciate ligament injury measured by computed tomography: aspects on using non-injured leg as control. BMC Musculoskelet Disord 2013; $14: 150$.

[50] Ardern C, Webster K, Taylor N, Feller J. Return to sport following anterior cruciate ligament reconstruction surgery: a systematic review and meta-analysis of the state of play. Br J Sports Med 2011;45:596.

[51] Gruber M, Gollhofer A. Impact of sensorimotor training on the rate of force development and neural activation. Eur J Appl Physiol 2004;92:98-105. 